\title{
A 3-Tier UWB-Based Indoor Localization System for Ultra-Low-Power Sensor Networks
}

\author{
Zheng Li, Student Member, IEEE, Wim Dehaene, Senior Member, IEEE, Georges Gielen, Fellow, IEEE
}

\begin{abstract}
We present a 3-tier UWB-based indoor localization system. It consists of a large number of energy-scavenging-based cost-effective transmit-only tags, a small number of batterypowered hubs as relay stations and a few base stations. This hierarchical scheme is driven by the energy available at each node. Localization is based on the arrival time of the UWB pulses at reference nodes. We describe how the coordinates and transmit time of a tag are determined and how the ambiguous solution is eliminated with the proper geometry of 4 reference nodes. We formulate where to place the hubs as an optimization problem. The localization performance of the system is investigated as a function of several parameters such as non-ideal hub placement, hub localization error and TOA error.
\end{abstract}

Index Terms-UWB, indoor localization, TOA, ultra low power.

\section{INTRODUCTION}

W IRELESS sensor networks (WSNs) are expected to penetrate our homes, factories, warehouses and other industrial and domestic environments. The growing popularity of WSNs provides a viable solution to indoor localization. By using small wireless sensor nodes (SNs) that can easily be distributed throughout an indoor environment, the location of a wearable SN can be determined. The major challenges for indoor localization systems are the cost, constrained energy and location accuracy. They have to be taken into account in the early stages of system design and especially in the choice of the wireless communication technique. Pulsed UWB communication seems to be the ideal candidate for this context due to its ultra-fine time resolution, robustness against multipath fading, low-cost architecture and low-power-consuming operation [1] [2].

Several low-power UWB-based indoor localization systems have been published [3] [4] [5]. The existing systems tried to prove the feasibility of UWB-based localization. Thus they focused on the device-level implementation and limited their performance evaluations to a small-scale setup (one unknown node, a few reference nodes). The deployment of reference nodes is random and their locations are assumed to be absolutely correct, which is not fully the case in reality.

In this paper, we focus on designing an efficient scheme driven by cost, size and energy constraints of SN and making proper decisions at higher levels of abstraction. The proposed 3-tier architecture enables the use of energy-scavenging-based, low-cost, ultra-low-power tags. This system can be extended to

Manuscript received May 2, 2008; revised August 12, 2008 and November 14, 2008; accepted February 23, 2009. The associate editor coordinating the review of this letter and approving it for publication was S. Affes.

The authors are with K.U.Leuven, Dept. Elektrotechniek ESAT-MICAS, Kasteelpark Arenberg 10, B-3001 LEUVEN, Belgium. Phone: +32 16328618 (e-mail: \{zheng.li, georges.gielen\}@esat.kuleuven.be).

Digital Object Identifier 10.1109/TWC.2009.080602 a large scale by adding extra relatively cheaper hubs instead of expensive base stations. The tag transmit time and 3D location can be determined if at least 4 hubs receive this tag signal, while the ambiguous solution is eliminated with the help of the proper geometry of 4 hubs. A method for optimizing the number and the placement of hubs is described. Different positioning algorithms are applied for different scenarios. Simulations are conducted to assess the influences of several nonidealities on the localization performance.

Section II gives a description of how the 3-tier localization system is conceived and motivates this system concept. Section III highlights positioning algorithms. Section IV describes the method for optimizing the number and placement of hubs. Section V presents the simulation results concerning the sensitivity of the overall localization performance to non-ideal hub placement, hub localization error and TOA error. Section VI concludes the paper.

\section{The Conceived 3-Tier System}

Tags must be low cost to allow placement onto a large number of moderate-cost objects. In addition, it is too expensive and impractical to replace batteries at regular intervals for a large quantity of tags. Thus we aim for tags that are autonomously powered. The scavenged energy can be from ambient heat, light, vibration RF [6] or other sources [7]; it all depends on the application. Our setup is completely open to the actual scavenging method used. All the operations of the tag can then be performed in burst mode once enough energy is harvested. For this the overall system must be conceived such that there is as little power consumption as possible in the tags.

In addition to accurate ranging capability of UWB, the UWB transmitter benefits from low complexity, low transmit power, loose linearity requirements and the ability to operate in a duty-cycled way. Thus its energy consumption is extremely low [8], which can meet the limits of energy scavenging. Furthermore, its very low hardware complexity offers the potential for a low-cost and highly integrated solution. Thus the tag is chosen to have a UWB transmitter only in our system. However, tags can not cooperate without a receiver. Uncoordinated parallel transmissions may interfere and lead to reception failure, especially if traffic intensity is high. This tradeoff can be addressed by randomizing the tag transmit time combined with a direct sequence/time hopping technique. Although most of the complexity of UWB communication is in the receiver, it can be implemented in an energy efficient way [9]. Moreover this asymmetric property is desirable in many sensor network applications, i.e. the information is transmitted from an energy-limited sensor node to a receiving node that is more relaxed in energy due to the battery. 
The Cramer-Rao lower bound (CRLB) on the unbiased delay estimate [10] indicates that TOA measurement is the most capable to exploit the large bandwidth of the UWB signal. It estimates the delay of the signal which defines the distance between the transmitter and the receiver. To obtain the tag locations, each tag $i$ must have a direct TOA estimate to at least three reference nodes (2D) or four reference nodes (3D). For cost reasons, an accurate time reference should be avoided in the tags. Tags and reference nodes are not synchronized to a common clock. As a result, the tag transmit time is unknown which turns the standard distance formula into:

$$
c^{2}\left(\hat{t_{j}}-t_{i}\right)^{2}=\left(x_{j}-x_{i}\right)^{2}+\left(y_{j}-y_{i}\right)^{2}+\left(z_{j}-z_{i}\right)^{2}
$$

where $c$ is the speed of light, $t_{i}$ is the tag transmit time, $\left(x_{i}, y_{i}, z_{i}\right)$ are the coordinates of the tag $i$ to be estimated, $\left(x_{j}\right.$, $\left.y_{j}, z_{j}\right)$ are the coordinates of the receiving reference node $j$, $\hat{t_{j}}$ is the measured receiving time of the $j$ th reference node commonly modeled as:

$$
\hat{t_{j}}=t_{j}+n_{j}+b_{j}
$$

where $t_{j}$ is the real receiving time, $n_{j}$ is the error caused by the Gaussian noise and $b_{j}$ is a non-negative bias introduced due to LOS blockage [11]. An extra receiving node is required to obtain $t_{i}$. This feature enables more applications which are often interested in not only the location but also the time of occurrence of a transmission, e.g. sensors in process control.

This 2-tier localization scheme would require many synchronized reference nodes (base stations) covering the large monitored area to receive the signals from all tags with only a limited radio range. It is too expensive to have a localization system like this. Therefore, secondary nodes (hubs) are introduced in our system to interface between a large number of tags and a limited number of base stations. This 3-tier system (see Fig. 1) thus consists of three levels:

- Tags: autonomously-powered UWB transmit-only devices attached to the unknown-location objects. Tags broadcast their ID packets and possible sensor data in burst mode once enough energy for the transmission is harvested. Data is modulated on to the pulses by means of BPSK. Every bit is replaced by a set of Ns pulses, with Ns the length of the spreading code. This spreading code is employed to increase the processing gain of the receiver, enable multi-user access and reduce the spectral spikes of the transmitted waveform.

- Hubs: battery-powered relay stations. Hubs collect and forward data from the transmit-only tag together with the time they receive this tag packet $\left(t_{r x}^{\prime}\right)$ and the time they transmit their packet $\left(t_{t x}^{\prime}\right)$ to base stations. Thanks to the low-power characteristic of UWB, the battery life of hubs can be very long (at least multiple months).

- Base stations: mains-powered computing devices. They are capable of performing all complex tasks, such as the calculation of tag locations and network management.

The differentiation in these three levels not only allows distributing complexity over separate blocks but also the energy consumption according to the energy source available at each level.

In this 3-tier system, 2-step calculation is performed to derive the tag's 3D location and its transmit time: first, the

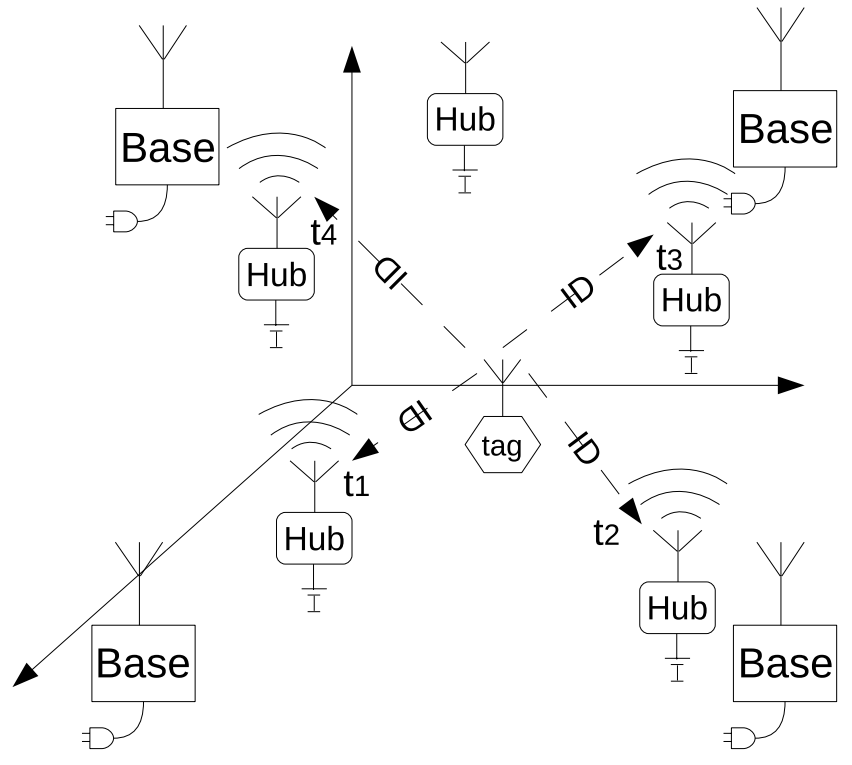

Fig. 1. A 3-tier wireless localization system with autonomously powered tags transmitting to hubs which transmit to base stations

location and transmit time $t_{t x}$ (which refers to the absolute time reference) of at least 4 hubs are solved; secondly, the tag location and its transmit time are calculated based on the information of these hubs. Clock synchronization between hubs is not required. By sending both $t_{r x}^{\prime}$ and $t_{t x}^{\prime}$ (which might refer to different time references for different hubs), the clock difference can be calibrated:

$$
t_{r x}=t_{t x}-t_{t x}^{\prime}+t_{r x}^{\prime}
$$

As such the arrival time of the tag signal at different hubs can be calibrated to the same time reference. Clock drift during the time of receiving and transmitting the signal may also introduce some errors. However, estimation can be performed [12] and this error can be compensated.

\section{Positioning Algorithm}

Location estimation involves solving (1) for $\left(x_{i}, y_{i}, z_{i}, t_{i}\right)$. We compared different positioning algorithms in [13]. For cost reasons, we aim to do localization based on a minimum number of reference nodes. For direct calculation, four hubs ${ }^{1}$ are required. According to the deployment of hubs (see Section IV), the probability of having more than 4 receiving hubs is quite high. In this scenario, a nonlinear optimization method has also been applied to achieve a higher location accuracy compared to the direct calculation method. The direct calculation and nonlinear optimization methods are now reviewed.

\section{A. Direct calculation}

The unknown location and transmit time are directly calculated by solving (1), where $j=1,2,3,4$. Three linear equations can be derived by subtracting the first equation from the others. It is thus possible to express 3 unknowns in terms of the fourth. Substitution in one of the original quadratic equations will then produce a quadratic equation in one

\footnotetext{
${ }^{1}$ Four hubs are located in four non-planar vertices of a cube.
} 
variable. As is well-known, solving this gives 2 solutions. [4] proposed to get rid of one solution $(x, y, z, t)$ with no physical meaning or beyond the monitored area. But according to our simulations, the chance of obtaining 2 possible physical solutions is quite high. A unique solution can be derived by adding one extra reference node but at a higher system cost.

The problem can still be solved with the proper geometry of 4 reference nodes. We consider a cubic area $\left(5 \times 5 \times 5 \mathrm{~m}^{3}\right)$ as an example in which the tag is situated. Simulations are conducted with different placements of 4 hubs: a) randomly located in the cube, b) on the surface of the cube, c) in the vertices of the cube. The whole cube is scanned with $10 \mathrm{~cm}$ resolution for the tag locations. The percentages of having two meaningful solutions are $26.02 \%, 14.56 \%, 0 \%$ for the aforementioned three placements. The results show that in a single-cube scenario the ambiguity can be eliminated by removing the solution with no physical meaning or beyond the cube area, when the 4 reference points are located in any 4 non-planar vertices of a cube. Therefore, the cube with 4 nonplanar reference nodes can be regarded as a basic cell, which is more practical than for instance a sphere. The monitored area can then be subdivided into many of these basic cells. The body diagonal of the cube, i.e. the longest distance a tag signal may travel in the cube, is then limited by the tag radio range. In the end, a tag in a large monitored area can either be received by 4 hubs or more than 4 hubs depending on the location of this tag. If more than 4 hubs receive this tag signal, a unique solution can be derived. If only 4 hubs receive this tag signal, the tag can only be inside the cube that these 4 hubs form. Thus the ambiguous solution can be dropped.

\section{B. Nonlinear optimization}

The location and transmit time can also be obtained by solving a weighted least squares problem. The objective function is defined as:

$$
\begin{gathered}
f(x, y, z, t)=\sum_{j=1}^{n} \beta_{j}\left(\left(\left(x_{i}-x_{j}\right)^{2}+\left(y_{i}-y_{j}\right)^{2}\right.\right. \\
\left.\left.+\left(z_{i}-z_{j}\right)^{2}\right)^{\frac{1}{2}}-c\left(t_{j}-t_{i}\right)\right)^{2}
\end{gathered}
$$

The weights $\beta_{j}$ can be used to characterize the reliability of each TOA measurement. Small weights are chosen for measurements with large errors and vice versa. If no reliability information is available, $\beta_{j}=1$ for all $j$. Clearly, the objective function is the summation of the squared range errors. The purpose is to find a solution $(x, y, z, t)$ that minimizes this objective function. Many algorithms are proposed to solve this problem. We use the Nelder-Mead simplex search method. Matlab provides a function based on this algorithm. We modified this function such that the lower and upper bound of the variables can be set, which results in a faster convergence and more accurate location estimation. The initial estimated location coordinates are chosen to be the mean location of the reference nodes. The initial estimated transmit time is chosen to be some time earlier than the earliest receiving time [4]. This reasonable initialization is capable to achieve the global minimum. This method requires at least 5 reference nodes. Its iterative nature requires large computational resources. Since a base station is plugged into the mains, it has plenty of energy and hence will be capable of performing such complex tasks.

\section{The Optimal Number and Placement of Hubs}

Hubs are introduced as secondary nodes. As discussed above, the total monitored area is subdivided into several cubes. Each cube must have at least four hubs in its vertices to make sure that tags anywhere in the room can be received by at least four hubs. The number of hubs must be minimized for cost reasons. Fortunately, sharing hubs between neighboring cubes allows to reduce the required number of hubs. There are many possibilities to allocate 4 hubs to each cube. Thus multiple hub placements can achieve the minimum number of hubs. [13] intuitively presents 2 different hub placements that both achieve this minimum. In some of these, however, hubs are more concentrated in the central area. This may cause more conflicts of signals in this hub-densely-located area. In order to maximally distribute hubs across the room and thus to minimize conflicts, the number of hubs in each cube should be the same as much as possible. We take the variance of the number of hubs in a cube as a measure of how evenly the hubs are distributed across the space:

$$
\sigma^{2}=\frac{1}{N} \sum_{i=1}^{N}\left(x_{i}-\bar{x}\right)^{2}
$$

where $N$ is the number of cubes in a room, $x_{i}$ is the number of hubs in the cube $i, \bar{x}$ is the mean of $x_{i}$. The goal is to minimize $\sigma^{2}$. The smaller this value, the more equal the number of hubs in each cube is. The hub placement can thus be achieved by solving a multi-objective optimization problem of the form:

$$
\begin{aligned}
& f\left(v_{i}\right)=\sum v_{i}+\text { weight } \times \sum c_{j} \\
& \text { such that } \quad c_{j} \geq 4 \& p_{k} \leq 3
\end{aligned}
$$

where $v_{i}$ has a binary value representing whether a hub is assigned to the vertex $i$ (1) or not $(0), c_{j}, p_{k}$ represent the number of hubs in the $j$ th cube and the $k$ th quadrangle respectively. The goal is to minimize this objective function $f\left(v_{i}\right)$. The first term in it is the total number of hubs. The second term is the simplified version of (5) with a small weight value. This weight makes the first term dominant. The simplified version of (5) is derived by setting $\bar{x}$ to a constant value (4 in our case) and turns it into a linear problem. The two constraints guarantee that there are at least 4 non-planar hubs in each cube. Matlab provides a function to solve this problem using a linear-programming-based branch-and-bound algorithm. Taking a $15 \times 15 \times 10 \mathrm{~m}^{3}$ room as an example, the number of hubs is minimized and the hubs are evenly distributed across the room (see Fig. 2) after applying method (6).

The minimum number of hubs is a function of the tag radio range $(R)$ and the room dimensions $(l, w, h)$. It is derived with a recursive process using the results from (6):

$$
\begin{aligned}
& \left.\# h u b(l, w, h, R)=\frac{Q}{2}\left(\frac{M}{2}(2 N+1)+\left[\frac{m+1}{2}\right]\left\lceil\frac{n}{2}\right)\right\rceil\right) \\
& +\left[\frac{q+1}{2}\right]\left\lceil\frac{m}{2}\right\rceil\left\lceil\frac{n}{2}\right\rceil \\
& N, M, Q=\left\{\begin{array}{lr}
n, m, q & n, m, q \text { even } \\
n+\frac{1}{2}, m+1, q+1 & n, m, q \text { odd }
\end{array}\right\}
\end{aligned}
$$

where $n, m, q=\left\lceil\frac{l, w, h}{R / \sqrt{3}}\right\rceil$ and $\lceil\cdot\rceil,[\cdot]$ denote the division ceiling operation and the remainder operation.

The formula (7) indicates how many more hubs are required for a larger space or a shorter tag radio range. For instance, 5 


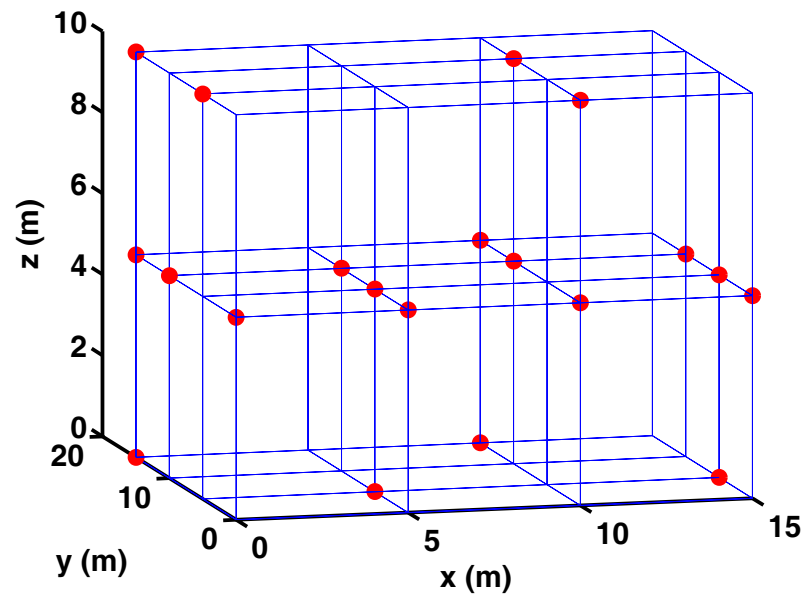

Fig. 2. The optimized hub placement in a $15 \times 15 \times 10 \mathrm{~m}^{3}$ room

hubs are needed for a $5 \times 10 \times 5 \mathrm{~m}^{3}$ room with a tag radio range of $5 \sqrt{3} \mathrm{~m}$, while a bigger $50 \times 10 \times 5 \mathrm{~m}^{3}$ room requires 26 hubs and this drops to 16 for a longer tag radio range of $15 \mathrm{~m}$. Hence, the proposed scheme is quite suitable for lowdata-rate systems, which can operate over significantly longer ranges indoors. That is because a low-data-rate system can utilize higher peak powers than those allowable for a highdata-rate system.

\section{IMPACTS OF NONIDEALITIES}

Matlab models have been developed to assess the impacts of several nonidealities on the localization performance. The simulation results are used to guide the hardware implementation of our system in practice.

\section{A. Non-ideal hub placement}

The hubs are located at some specific locations (the corners of the cubes) to achieve the minimum numbers and avoid ambiguities in the solutions. Since in a realistic environment it is often difficult to have a complete freedom of determining hub locations (e.g. attach the hubs to racks), the sensitivity of the localization performance to non-ideal hub placement has been investigated. A monitored area $\left(5 \times 10 \times 5 \mathrm{~m}^{3}\right)$ is scanned with $50 \mathrm{~cm}$ resolution for the tag locations. A hub placement deviation is the maximum distance that the hub can be located away from its optimal location. At each test hub placement deviation, we then conduct 1000 runs of MonteCarlo simulation with all hubs randomly located around their ideal locations. If the tag either can not be seen by 4 hubs or its ambiguity can not be eliminated, it is considered an undetectable tag. The failure rates are averaged over these 1000 runs. The results depicted in Fig. 3 show that although the failure rate is small for a tag radio range of $5 \sqrt{3} m$ (the same as the cell radius), zero failure rate is only achievable when the hubs are in their optimal locations. One possible solution is to use a cell radius smaller than the tag radio range. To illustrate this, the tag radio range is set to $10 \mathrm{~m}$, while the cube diagonal is still $5 \sqrt{3} \mathrm{~m}$. The failure detection rate of tags dramatically drops (see Fig. 3). In this scenario, hubs can

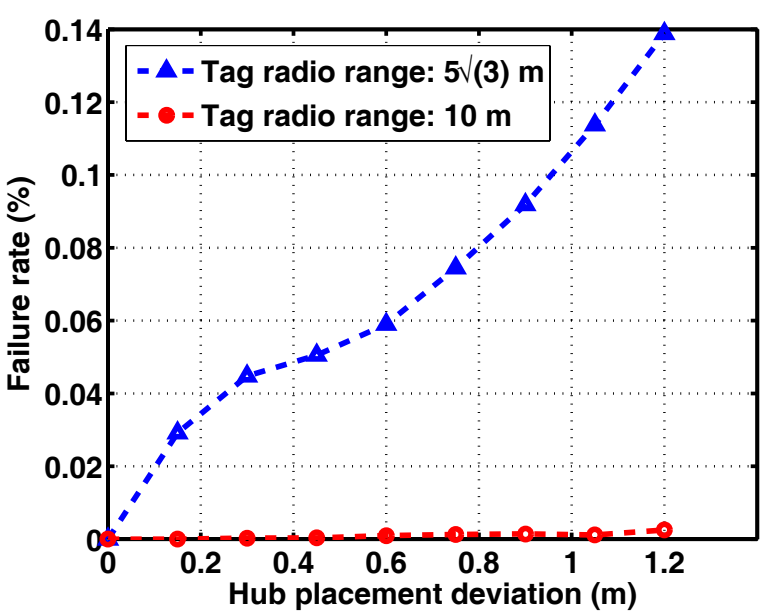

Fig. 3. The failure detection rate of tags versus the deviation of hub placement, which is the maximum distance that a hub can be located from its ideal location in a $5 \times 10 \times 5 \mathrm{~m}^{3}$ room with cell radius $5 \sqrt{3} \mathrm{~m}$

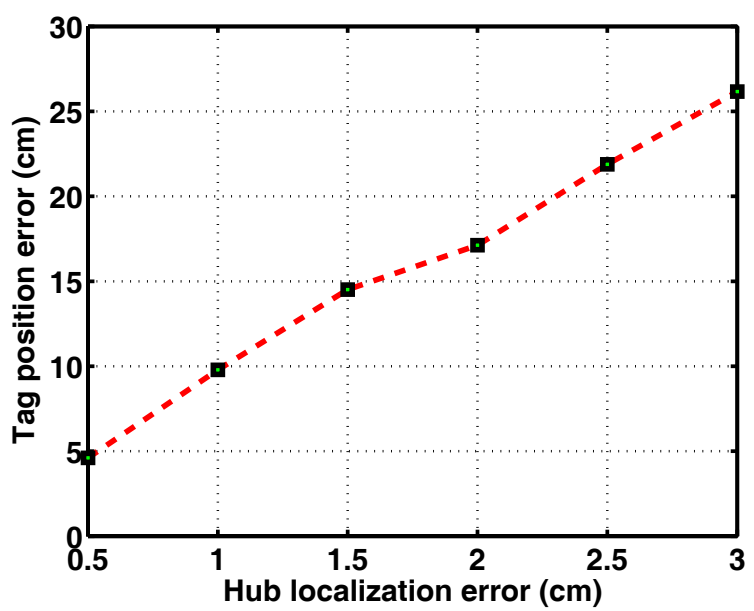

Fig. 4. The tag location accuracy versus the hub localization error in a $5 \times 5 \times 5 \mathrm{~m}^{3}$ cubic area

be located furthest $45 \mathrm{~cm}$ away from their optimal locations before some tags become undetectable. That is to say that all the tags can be detected for a hub placement error of $5 \%$ of the cell radius if the tag radio range is about 1.2 times larger than the cell radius.

\section{B. Hub localization error}

The hub locations are first derived and subsequently used to localize the tags. Thus any hub localization error which leads to uncertainty in the hub locations $\left(x_{j}+\Delta x_{j}, y_{j}+\Delta y_{j}\right.$, $z_{j}+\Delta z_{j}$ ) will obviously result in an unprecise tag location estimation. A cubic area $\left(5 \times 5 \times 5 \mathrm{~m}^{3}\right)$ is scanned with 50 $\mathrm{cm}$ resolution for the tag locations. In Monte-Carlo simulation 1000 random samples of hub locations are generated for a given hub localization error. Fig. 4 depicts the relationship between the worst-case tag location accuracy and the hub localization error. It clearly shows that in order to achieve a target $10 \mathrm{~cm}$ location accuracy of the tag, a $1 \mathrm{~cm}$ hub location accuracy must be guaranteed in the hub localization. 


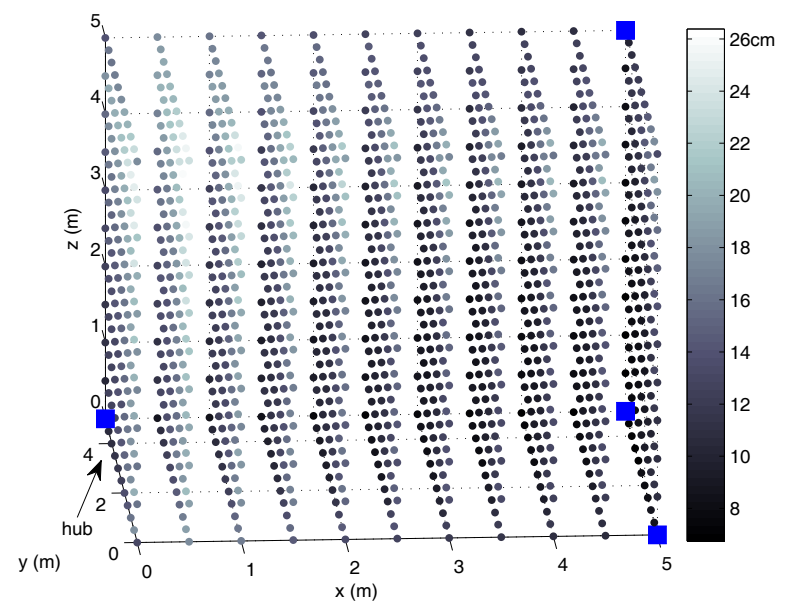

Fig. 5. Tag location error averaged over 1000 runs for a $\sigma$ (TOA error) of $200 \mathrm{ps}$ in a $5 \times 5 \times 5 \mathrm{~m}^{3}$ cube. The colors in grayscale indicate the location error of every tag

\section{TOA error}

The positioning algorithms described in Section III use the arrival time of UWB pulses. Therefore, the TOA measurement accuracy influences the location accuracy. Such TOA error may be caused by imperfect synchronization, timing errors (clock jitter), additive white Gaussian noise, multipath and non-line-of-sight (NLOS) propagation. In our 2-step location calculation, the hub locations are static and determined once for any given configuration. Averaging over time can be used to improve the accuracy of the hub location. As a starting point, we assume that the first-step (hub to base station) TOA measurements are accurate: we can solve accurately the hub transmit time and 3D location. The tag location accuracy is now evaluated by considering that the second-step TOA measurements (tag to hub) can be erroneous. In this paper we consider LOS conditions and model this TOA error as a Gaussian random variable. Assume that the erroneous TOA at the $j$ th hub is $t_{j}+\Delta t_{j}$, where the $\Delta t_{j}$ is a random variable of normal distribution $\left(N\left(0, \sigma^{2}\right)\right)$. In Monte-Carlo simulations, 1000 random samples of $N\left(0, \sigma^{2}\right)$ are generated for a given $\sigma$ (the standard deviation of the TOA error).

The geometrical constellation of hubs and tags influences the accuracy of the location estimation. In our experiments, a cubic area $\left(5 \times 5 \times 5 \mathrm{~m}^{3}\right)$ is scanned with $50 \mathrm{~cm}$ resolution for the tag locations. Each tag location is averaged over 1000 runs with a $\sigma$ (TOA error) of $200 \mathrm{ps}$. Their location errors are shown in Fig. 5. We can clearly see that the TOA error is not equally translated to the location errors at all locations of the area.

The tag location error varies among 1000 random samples of $N\left(0, \sigma^{2}\right)$. Since tags are not stationary but can move in certain applications, averaging may not help to improve the location accuracy. In order to make sure that every localization will achieve a certain target accuracy (for example $10 \mathrm{~cm}$ ), we require for every tag location that $\geq 99.7 \%$ out of 1000 runs with random TOA error satisfy a location error $\leq 10 \mathrm{~cm}$. Fig. 6 depicts the percentage of tags that satisfy with $\geq 99.7 \%$ the $10 \mathrm{~cm}$ location accuracy versus the $\sigma$ (TOA error) in a cubic

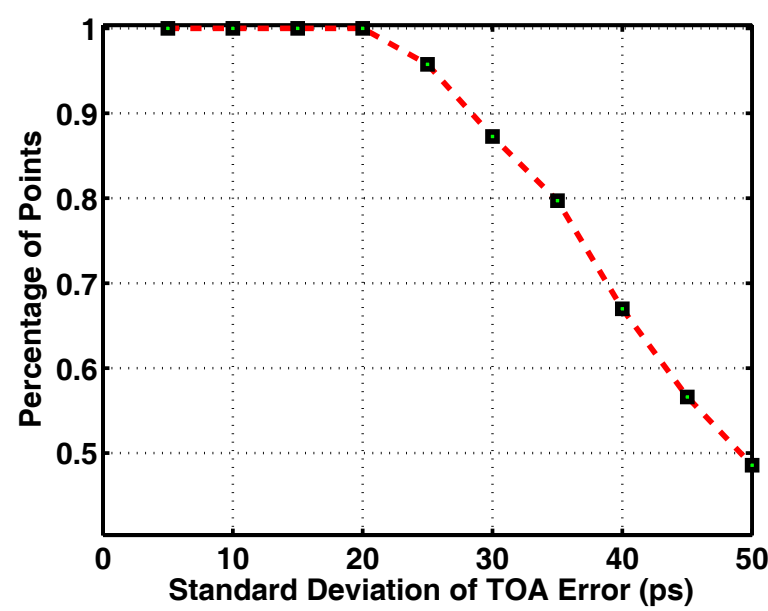

Fig. 6. The percentage of tags that satisfy with $\geq 99.7 \%$ the $10 \mathrm{~cm}$ location accuracy as a function of the $\sigma$ (TOA error) in a $5 \times 5 \times 5 \mathrm{~m}^{3}$ cube

area. It clearly shows that $\sigma$ (TOA error) must be $\leq 20 \mathrm{ps}$ to achieve a $10 \mathrm{~cm}$ location accuracy in a whole cubic area. It is quite challenging to achieve less than 20 ps TOA jitter using a $1 \mathrm{GHz}$ bandwidth with 1 pulse. Fortunately, averaging over several received pulses helps to improve TOA accuracy and can easily be done since one bit is multiple pulses.

\section{CONCLUSION}

In this paper, the design of a 3-tier UWB-based indoor localization system has been described from a system perspective. In order to make tags low cost, small size and ultra low power, they are autonomously powered and designed with only UWB transmitters for communication. Adding relatively cheaper hubs as intermediate relay stations between transmitonly tags and base stations makes the system more practical with low cost. The tag's 3D location and transmit time can be derived with the proper geometry of 4 hubs. A method to determine the minimal number and the optimal placement of hubs in a given room has been proposed. We have assessed the impact of hub placement error, hub localization error and TOA error on the localization performance. We have concluded that making the cell radius smaller than the tag radio range helps to alleviate the effect of hub placement error. In order to achieve a $10 \mathrm{~cm}$ location accuracy of the tag, a $1 \mathrm{~cm}$ hub location accuracy must be guaranteed in the hub localization and the $\sigma$ (TOA error) must be $\leq 20 \mathrm{ps}$. These results have been used to guide the hardware design of the system.

\section{ACKNOWLEDGMENT}

The authors acknowledge the support of IWT under the Pinballs SBO project.

\section{REFERENCES}

[1] D. Porcino and W. Hirt, "Ultra-wideband radio technology: potential and challenges ahead," IEEE Commun. Mag., 2003.

[2] S. Gezici et al., "Localization via ultra-wideband radios: a look at positioning aspects for future sensor networks," IEEE Signal Processing Mag., vol. 22, 2005.

[3] R. J. Fontana et al., "Commercialization of an ultra wideband precision asset location system," in Proc. IEEE Conf. UWBST, 2003. 
[4] K. Yu and I. Oppermann, "Performance of UWB position estimation based on time-of-arrival measurements," in Proc. IEEE Conf. UWBST, 2004.

[5] G. Zhang and S. V. Rao, "Position localization with impulse ultra wide band," in Proc. IEEE/ACES Conf. Wireless Commun. Applied Computational Electromagnetics, 2005.

[6] M. Baghaei-Nejad, Z. Zou, H. Tenhunen, and L. R. Zheng, "A novel passive tag with asymmetric wireless link for RFID and WSN applications," in Proc. IEEE Circuits Systems, 2007.

[7] P. Fiorini, I. Doms, C. van Hoof, and R. Vullers, "Micropower energy scavenging," in Proc. ESSCIRC, 2008.

[8] J. Ryckaert et al., "0.65-to-1.4nJ/burst 3-to-10GHz UWB digital TX in 90nm CMOS for IEEE 802.15.4a," in Proc. IEEE ISSCC, 2007.
[9] M. Verhelst, N. Van Helleputt, G. Gielen, and W. Dehaene, "A reconfigurable, $0.13 \mu \mathrm{m}$ CMOS $110 \mathrm{pJ} /$ pulse, fully integrated IR-UWB receiver for communication and sub-cm ranging," in Proc. IEEE ISSCC, 2009.

[10] H. V. Poor, An Introduction to Signal Detection and Estimation. Springer, pp. 331-333, 1994.

[11] I. Guvenc, C.-C. Chong, and F. Watanabe, "NLOS identification and mitigation for UWB localization systems," in Proc. IEEE WCNC, 2007.

[12] A. Wellig and Y. Qiu, "Trellis-based maximum-likelihood crystal drift estimator for ranging applications in UWB-LDR," in Proc. IEEE Conf. UWB, 2006.

[13] Z. Li, W. Dehaene, and G. Gielen, "System design for ultra-low-power UWB-based indoor localization," in Proc. IEEE Conf. UWB, 2007. 